\title{
ON COUPLED INTEGRAL H-LIKE EQUATIONS OF CHANDRASEKHAR*
}

\author{
JONQ JUANG ${ }^{\dagger}$
}

\begin{abstract}
A recently proposed "simple transport model" equation with an "angular shift" $\alpha \quad(0 \leq \alpha \leq 1)$ leads to a coupled integral H-like equation of Chandrasekhar. Such coupled H-like equations can be treated in terms of a one-parameter $\left(k_{1}, 0<k_{1}<1\right)$ family. From there an a priori bound can be obtained, which is independent of $k_{1}, \alpha$, and $c \quad(0 \leq c \leq 1)$. Here $c$ denotes the average total number of particles emerging from a collision. Consequently, we conclude that positive solutions of such coupled integral H-like equations exist. Moreover, we show that such equations have a unique positive solution pair for $c=0$ or $c=1$ and $\alpha=0$ or $\alpha=1$, and that the equations have exactly two positive solution pairs for $0<c<1$ and $0 \leq \alpha<1$ or $c=1$ and $\alpha$ sufficiently close to 1 .
\end{abstract}

Key words. integral equation, H-like functions of Chandrasekhar, a priori bound, existence and multiplicity

AMS subject classifications. $45 \mathrm{G} 10,82 \mathrm{C} 70,85 \mathrm{~A} 25$

1. Introduction. In this work we study the coupled integral H-like equations of the form

$$
H_{1}(\mu)=1+\frac{c}{2} H_{1}(\mu)(\mu+\alpha) \int_{\alpha}^{1} \frac{H_{2}\left(\mu^{\prime \prime}\right)}{\mu+\mu^{\prime \prime}} d \mu^{\prime \prime},-\alpha \leq \mu \leq 1,
$$

and

$$
H_{2}\left(\mu^{\prime}\right)=1+\frac{c}{2} H_{2}\left(\mu^{\prime}\right)\left(\mu^{\prime}-\alpha\right) \int_{-\alpha}^{1} \frac{H_{1}\left(\mu^{\prime \prime}\right)}{\mu^{\prime}+\mu^{\prime \prime}} d \mu^{\prime \prime}, \alpha \leq \mu^{\prime} \leq 1 .
$$

Here $c$ denotes the average total number of particles emerging from a collision, which is assumed to be conservative, i.e., $c \leq 1$, and $\alpha$ denotes an "angular shift" with $0 \leq \alpha \leq 1$. Equation (1) first appeared in [8], where it was derived from a "simple transport model" (see, e.g., [5], [8]) using Chandrasekhar's method of solution. For $\alpha=0$, equation (1) reduces to Chandrasekhar's well-known integral equation. Various methods (see, e.g., [1]-[4], [6], [7], [10], [11]) have been applied to such equations. In summary, they have shown that Chandrasekhar's integral equation has one solution if $c=1$ and at most two solutions if $c<1$.

In this article, we first show that an a priori bound, which is independent of $c$ and $\alpha$, can be obtained by introducing a one-parameter $\left(k_{1}, 0<k_{1}<1\right)$ family. Therefore, the degree theory is applied to show the existence of positive solutions. Second, the techniques used in [6], [10] are generalized to show that equation (1) has a unique positive solution pair for $c=0$ or $c=1$ and $\alpha=0$ or $\alpha=1$, and that equation (1) has exactly two positive solution pairs for $0<c<1$ and $0 \leq \alpha<1$ or $c=1$ and $\alpha$ sufficiently close to 1 . The above results are contained in $\S 2$.

We conclude this introductory section by noting that using the solutions obtained by equation (1), the simple transport model can then be treated as a "pure" initial

* Received by the editors June 15, 1993; accepted for publication (in revised form) December 15, 1993. This research was partially supported by the National Science Council of the Republic of China.

† Department of Applied Mathematics, National Chiao Tung University, Hsinchu, Taiwan, Republic of China. 
value problem. More precisely, consider the following simple transport model:

$$
\begin{aligned}
(\mu+\alpha) \frac{\partial \phi(x, \mu)}{\partial x}+\phi(x, \mu) & =\frac{c}{2} \int_{-1}^{1} \phi\left(x, \mu^{\prime}\right) d \mu^{\prime}, 0 \leq x<\infty,|\mu| \leq 1 \\
\phi(0, \mu) & =f(\mu), 1 \geq \mu>-\alpha .
\end{aligned}
$$

Then, for $-1 \leq \mu \leq-\alpha$, we have (see equations (3) and (12) of [8]) that

$$
\phi(0, \mu)=\frac{c}{2} \int_{-\alpha}^{1} \frac{\mu^{\prime}+\alpha}{\mu^{\prime}-\mu} H_{1}\left(\mu^{\prime}\right) H_{2}(-\mu) f\left(\mu^{\prime}\right) d \mu^{\prime} .
$$

Such an approach provides an interesting and effective alternative for solving the simple transport model theorectically as well as numerically.

2. Main results.

Notation. Set

$$
\begin{aligned}
& x=\frac{c}{2} \int_{-\alpha}^{1} H_{1}(\mu) d \mu, \quad y=\frac{c}{2} \int_{\alpha}^{1} H_{2}\left(\mu^{\prime}\right) d \mu^{\prime}, \\
& a=\frac{c^{2}}{4} \int_{-\alpha}^{1} \int_{\alpha}^{1} H_{1}(\mu) H_{2}\left(\mu^{\prime \prime}\right) \frac{\mu^{\prime \prime}-\alpha}{\mu+\mu^{\prime \prime}} d \mu^{\prime \prime} d \mu,
\end{aligned}
$$

and

$$
b=\frac{c^{2}}{4} \int_{-\alpha}^{1} \int_{\alpha}^{1} H_{1}(\mu) H_{2}\left(\mu^{\prime \prime}\right) \frac{\mu+\alpha}{\mu+\mu^{\prime \prime}} d \mu^{\prime \prime} d \mu .
$$

Note that $a+b=x y$. We begin by deriving some integral properties which a solution of (1) must satisfy.

LEMMA 1. If $H_{1}$ and $H_{2}$ are solutions of (1), then the following holds:

$$
(1-x)(1-y)=1-c \text {. }
$$

Proof. Multiplying equation (1) by $\frac{c}{2}$ and integrating equations (1a) and (1b) over the ranges of $\mu^{\prime}$ and $\mu$, respectively, we obtain

$$
x=\frac{c}{2}(1+\alpha)+x y-a
$$

and

$$
y=\frac{c}{2}(1-\alpha)+x y-b .
$$

Adding up (3a) and (3b) would yield the assertion of Lemma 1.

Remark. For $\alpha=0,(2)$ reduces to some well-known expressions concerning the properties of $H$ equations (see, e.g., [3, pp. 106-107]).

For $\alpha \neq 1$, we see immediately that if $H_{1}$ and $H_{2}$ are positive solutions of (1), then there must exist two positive numbers $k_{1}$ and $k_{2}$, where $0<k_{1}, k_{2}<1$ and $k_{1}+k_{2}=1$, so that

$$
a=k_{1} x y
$$

and

$$
b=k_{2} x y \text {. }
$$


It then follows from (2), (3), and (4) that the following holds:

$$
\begin{aligned}
& x=\frac{1-\frac{c}{2}(1-\alpha)+k_{1} c \pm \sqrt{\left[1-\frac{c}{2}(1-\alpha)+k_{1} c\right]^{2}-2 k_{1}(1+\alpha) c}}{2 k_{1}}:=a_{1} \pm b_{1}, \\
& y=\frac{1-\frac{c}{2}(1+\alpha)+k_{2} c \pm \sqrt{\left[1-\frac{c}{2}(1+\alpha)+k_{2} c\right]^{2}-2 k_{2}(1-\alpha) c}}{2 k_{2}}:=a_{2} \pm b_{2} .
\end{aligned}
$$

Since $k_{1}$ and $k_{2}$ are to be treated as real parameters, necessary conditions for (5) to be meaningful are that both $\left[1-\frac{c}{2}(1-\alpha)+k_{1} c\right]^{2}-2 k_{1} c(1+\alpha)$ and $\left[1-\frac{c}{2}(1+\alpha)+\right.$ $\left.k_{2} c\right]^{2}-2 k_{2} c(1-\alpha)$ are nonnegative. However, these are so if $0 \leq \alpha \leq 1$ and $0 \leq c \leq 1$. To see this, we note that, for $c \neq 0, f_{1}\left(k_{1}\right):=\left[1-\frac{c}{2}(1-\alpha)+k_{1} c\right]^{2}-2 k_{1} c(1+\alpha)$ has a minimim $(1+\alpha)(1-\alpha)(1-c)$, which is nonnegative whenever $0 \leq \alpha \leq 1$ and $0 \leq c \leq 1$.

We denote by $S$ the feasible region $\{(k, c, \alpha): 0<k<1,0 \leq c \leq 1$ and $0 \leq$ $\alpha \leq 1\}$ for the solution of (1). The cross-section $\{(k, c, \alpha): 0<k<1,0 \leq c \leq$ 1 and $\alpha$ is fixed $\}$ of $S$ will be denoted by $S_{\alpha}$. The properties and signs of $1-x$ and $1-y$ will be examined in the next lemmas.

LEMMA 2. (i) $1-a_{1}+b_{1} \geq 0$ and $1-a_{1}-b_{1} \leq 0$ for all $\left(k_{1}, c, \alpha\right) \in S$.

(ii) $1-a_{2}+b_{2} \geq 0$ and $1-a_{2}-b_{2} \leq 0$ for all $\left(k_{2}, c, \alpha\right) \in S$.

(iii) For each fixed $\alpha$, where $0 \leq \alpha<1$, we have that $1-a_{1}+b_{1}$ and $1-a_{2}+b_{2}$, considered as functions from $S_{\alpha} \rightarrow R$, can be continuously extended to $\bar{S}_{\alpha}$.

(iv) Let $c$ be sufficiently small, say $0 \leq c \leq \frac{1}{8}$. Then $1-a_{1}+b_{1} \geq \frac{1}{2}$ and $1-a_{2}+b_{2} \geq \frac{6}{7}$ for all $k_{1}$ and $k_{2}, 0<k_{1}, k_{2}<1$, and all $\alpha, 0 \leq \alpha \leq 1$.

Proof. Since the computation leading to (i) and (ii) is similar, we shall only illustrate (i). To see (i), it suffices to show that $b_{1}^{2} \geq\left(1-a_{1}\right)^{2}$, or equivalently

$$
\left[1-\frac{c}{2}(1-\alpha)+k_{1} c\right]^{2}-2 k_{1} c(1+\alpha)-\left[\left(2 k_{1}-1\right)\left(1-\frac{c}{2}\right)-\frac{c \alpha}{2}\right]^{2} \geq 0 .
$$

Since the left-hand side of the inequality is equal to $4\left(1-k_{1}\right)\left(k_{1}\right)(1-c)$, the assertion of Lemma 2(i) thus follows. To prove (iii), we note that

$$
a_{1}-b_{1}=\frac{(1+\alpha) c}{1-\frac{c}{2}(1-\alpha)+k_{1} c+\sqrt{\left[1-\frac{c}{2}(1-\alpha)+k_{1} c\right]^{2}-2 k_{1} c(1+\alpha)}}:=\frac{(1+\alpha) c}{g_{1}\left(k_{1}, c, \alpha\right)}
$$

and

$$
a_{2}-b_{2}=\frac{(1-\alpha) c}{1-\frac{c}{2}(1+\alpha)+k_{2} c+\sqrt{\left[1-\frac{c}{2}(1+\alpha)+k_{2} c\right]^{2}-2 k_{2} c(1-\alpha)}}:=\frac{(1-\alpha) c}{g_{2}\left(k_{2}, c, \alpha\right)} .
$$

Since $g_{1}\left(k_{1}, c, \alpha\right) \geq \frac{1}{2}$ for all $\left(k_{1}, c, \alpha\right) \in \bar{S}$, we conclude that $a_{1}-b_{1}$, and hence $1-a_{1}+b_{1}$, can be continuously extended to $\bar{S}$. Now, if $\alpha$ is fixed as assumed, then $g_{2}\left(k_{2}, c, \alpha\right) \geq \frac{1}{2}(1-\alpha)>0$ for all $\left(k_{2}, c\right)$. Therefore, for each fixed $\alpha, 1-a_{2}+b_{2}$ can be continuously extended to $\bar{S}_{\alpha}$.

To prove (iv), we see that if $0 \leq c \leq \frac{1}{8}$, then $a_{1}-b_{1}=\frac{(1+\alpha) c}{g_{1}\left(k_{1}, c, \alpha\right)} \leq 2 c(1+\alpha) \leq \frac{1}{2}$, for all $k_{1}$ and $\alpha$. Thus, $1-a_{1}+b_{1} \geq \frac{1}{2}$ as asserted. Similarly, we have

$$
a_{2}-b_{2}=\frac{(1-\alpha) c}{g_{2}\left(k_{2}, c, \alpha\right)} \leq \frac{c}{1-\frac{c}{2}(1+\alpha)} \leq \frac{c}{1-c} \leq \frac{1}{7} \text {. }
$$

Therefore, $1-a_{2}+b_{2} \geq \frac{6}{7}$ as asserted. 
Remarks. The function $1-a_{1}+b_{1}$, as indicated in the proof, can be continuously extended to $\bar{S}$. However, the same assertion fails for $1-a_{2}+b_{2}$. To see this, we note that if $\alpha=1$, then $a_{2}-b_{2}=0$ for all $k_{2}$ and $c$. However, if $c=1$ then $a_{2}-b_{2}=1$ for any $\alpha(\neq 1)$ and $k_{2} \leq \frac{1}{2}(1-\alpha)$.

In view of (2), the case $c=1$ shall be further studied.

LEMmA 3. (i) $1-a_{1}+b_{1}=0$ if and only if $\frac{1}{2}(1+\alpha) \geq k_{1}$ and $c=1$. Moreover, $1-a_{1}-b_{1}=0$ if and only if $\frac{1}{2}(1+\alpha) \leq k_{1}$ and $c=1$.

(ii) $1-a_{2}+b_{2}=0$ if and only if $k_{1} \geq \frac{1}{2}(1+\alpha)$ and $c=1$. Furthermore, $1-a_{2}-b_{2}=0$ if and only if $k_{1} \leq \frac{1}{2}(1+\alpha)$ and $c=1$.

(iii) If $\frac{1}{2}(1+\alpha)<k_{1}$ and $c=1$, then $1-a_{1}+b_{1}=\frac{2 k_{1}-1-\alpha}{2 k_{1}}$. Moreover, if $\frac{1}{2}(1+\alpha)>k_{1}$ and $c=1$, then $1-a_{1}-b_{1}=\frac{2 k_{1}-1-\alpha}{2 k_{1}}$.

(iv) If $k_{1}<\frac{1}{2}(1+\alpha)$ and $c=1$, then $1-a_{2}+b_{2}=\frac{1+\alpha-2 k_{1}}{2\left(1-k_{1}\right)}$. Furthermore, if $k_{1}>\frac{1}{2}(1+\alpha)$ and $c=1$, then $1-a_{2}-b_{2}=\frac{1+\alpha-2 k_{1}}{2\left(1-k_{1}\right)}$.

Proof. The necessary parts of Lemma 3(i) follow from (2) and some simple algebra. The remainder of the proof is trivial and thus omitted.

Some simple algebra would yield the following equivalent formulation of (1).

LEMmA 4. The functions $H_{1}$ and $H_{2}$ satisfy, respectively,

$$
\left[H_{1}(\mu)\right]^{-1}=(1-y)+\frac{c}{2} \int_{\alpha}^{1} \frac{\mu^{\prime \prime}-\alpha}{\mu+\mu^{\prime \prime}} H_{2}\left(\mu^{\prime \prime}\right) d \mu^{\prime \prime}
$$

and

$$
\left[H_{2}\left(\mu^{\prime}\right)\right]^{-1}=(1-x)+\frac{c}{2} \int_{-\alpha}^{1} \frac{\mu^{\prime \prime}+\alpha}{\mu^{\prime}+\mu^{\prime \prime}} H_{1}\left(\mu^{\prime \prime}\right) d \mu^{\prime \prime}
$$

if and only if $H_{1}$ and $H_{2}$ satisfy (1a) and (1b), respectively.

In view of (2), we see that if $H_{1}$ and $H_{2}$ are solutions of (1), then either

$$
1-x \geq 0 \text { and } 1-y \geq 0
$$

or

$$
1-x \leq 0 \text { and } 1-y \leq 0 .
$$

Let $C[-\alpha, 1] \times C[\alpha, 1]$ be the Banach space of pairs of bounded real-valued continuous functions with sup norm. That is, if $\left(h_{1}, h_{2}\right) \in C[-\alpha, 1] \times C[\alpha, 1]$, then

$$
\left\|\left(h_{1}, h_{2}\right)\right\|_{\infty}:=\max \left\{\max _{-\alpha \leq \mu \leq 1}\left|h_{1}(\mu)\right|:=\left\|h_{1}\right\|_{\infty}, \max _{\alpha \leq \mu \leq 1}\left|h_{2}\left(\mu^{\prime}\right)\right|:=\left\|h_{2}\right\|_{\infty}\right\} .
$$

In preparation for the use of a homotopy invariance argument define, for $\left(K_{1}, K_{2}\right) \in$ $C[-\alpha, 1] \times C[\alpha, 1]$,

$$
\begin{gathered}
\psi_{1, c}\left(K_{2}(\mu)\right)=(1-y)+\frac{c}{2} \int_{\alpha}^{1} \frac{\mu^{\prime \prime}-\alpha}{\mu+\mu^{\prime \prime}} \frac{1}{K_{2}\left(\mu^{\prime \prime}\right)} d \mu^{\prime \prime} \\
\psi_{2, c}\left(K_{1}\left(\mu^{\prime}\right)\right)=(1-x)+\frac{c}{2} \int_{-\alpha}^{1} \frac{\mu^{\prime \prime}+\alpha}{\mu^{\prime}+\mu^{\prime \prime}} \frac{1}{K_{1}\left(\mu^{\prime \prime}\right)} d \mu^{\prime \prime} \\
\psi_{c}\left(K_{1}(\mu), K_{2}\left(\mu^{\prime}\right)\right)=\left(\psi_{1, c}\left(K_{2}(\mu)\right)\right), \psi_{2, c}\left(K_{1}\left(\mu^{\prime}\right)\right) .
\end{gathered}
$$

An a priori bound, which is independent of $k_{1}$ and $c$, is obtained in the following lemma. 
LemMA 5. Let $K_{1}$ and $K_{2}$ be any positive continuous solutions of $\left(K_{1}, K_{2}\right)=$ $\psi_{c}\left(K_{1}, K_{2}\right)$ satisfying (7a). Then there is an $m>0$ (independent of $c$ and $\alpha$ ) such that $K_{1}(\mu) \geq m$ and $K_{2}\left(\mu^{\prime}\right) \geq m$ for all $\left(\mu, \mu^{\prime}\right) \in[-\alpha, 1] \times[\alpha, 1]$, all $0 \leq \alpha \leq 1$, and all $0 \leq c \leq 1$.

Proof. Clearly, $H_{1}=\frac{1}{K_{1}}$ and $H_{2}=\frac{1}{K_{2}}$ are positive solutions of (1). Consequently, $1 \geq K_{1}(\mu)$ and $1 \geq K_{2}\left(\mu^{\prime}\right)$ for all $\left[\mu, \mu^{\prime}\right) \in[-\alpha, 1] \times[\alpha, 1]$. Therefore,

$$
K_{1}(\mu) \geq 1-y+\frac{c}{2} \int_{\alpha}^{1} \frac{\mu^{\prime \prime}-\alpha}{\mu^{\prime \prime}+1} d \mu^{\prime \prime}:=1-y+g_{1}(c, \alpha)
$$

and

$$
K_{2}\left(\mu^{\prime}\right) \geq 1-x+\frac{c}{2} \int_{-\alpha}^{1} \frac{\mu^{\prime \prime}+\alpha}{\mu^{\prime \prime}+1} d \mu^{\prime \prime}:=1-x+g_{2}(c, \alpha) .
$$

Since $1-y \geq 0$ and $1-x \geq 0$, there must exist positive constants $k_{1}$ and $k_{2}, k_{1}+k_{2}=1$, such that $1-x=1-a_{1}+b_{1}$ and $1-y=1-a_{2}+b_{2}$, where $a_{1}-b_{1}$ and $a_{2}-b_{2}$ are defined as in (5). Now, via Lemma 2(iv), $1-x \geq \frac{1}{2}$ for $0 \leq c \leq \frac{1}{8}$. Since, for fixed $c, g_{2}(c, \alpha)$ is an increasing function (in $\alpha$ ), we have that $\int_{-\alpha}^{1} \frac{\mu^{\prime \prime}+\alpha}{\mu^{\prime \prime}+1} d \mu^{\prime \prime} \geq 1-\ell n 2$. Consequently,

$$
K_{2} \geq \min \left\{\frac{1}{2}, \frac{1}{16}(1-\ell n 2)\right\}=\frac{1}{16}(1-\ell n 2):=m_{2}
$$

for all $\left(\mu, \mu^{\prime}\right) \in[-\alpha, 1] \times[\alpha, 1]$, all $0 \leq c \leq 1$, and all $0 \leq \alpha \leq 1$. On the other hand,

$$
y=\frac{c}{2} \int_{\alpha}^{1} \frac{1}{K_{2}\left(\mu^{\prime}\right)} d \mu^{\prime} \leq \frac{c(1-\alpha)}{2 m_{2}}
$$

and so $1-y \geq 1-\frac{c(1-\alpha)}{2 m_{2}}$. Hence, if $0 \leq c \leq m_{2}$ or $\alpha \geq 1-m_{2}$, then $1-y \geq \frac{1}{2}$. However, if $1 \geq c \geq m_{2}$ and $0 \leq \alpha \leq 1-m_{2}$ then

$$
g_{1}(c, \alpha) \geq \frac{m_{2}}{2} \int_{1-m_{2}}^{1} \frac{\mu^{\prime \prime}-\left(1-m_{2}\right)}{\mu^{\prime \prime}+1} d \mu^{\prime \prime}:=\bar{m}_{1}>0 .
$$

Consequently, $K_{1}(\mu) \geq \min \left\{\frac{1}{2}, \bar{m}_{1}\right\}:=m_{1}$ as asserted. The assertion of the lemma now follows by choosing $m=\min \left\{m_{1}, m_{2}\right\}$.

Remark. The lower bound for $K_{2}$ is not sharp. A better bound can be obtained. To see this, let $c$ be such that $0 \leq c \leq \frac{2}{9-\ell n 2}$, then $a_{1}-b_{1} \leq 2 c(1+\alpha) \leq \frac{8}{9 \ell n 2}$. Thus, $1-a_{1}+b_{1} \geq \frac{1-\ell n 2}{9-\ell n 2}$ for $0 \leq c \leq \frac{2}{9-\ell n 2}$. Hence,

$$
K_{2}\left(\mu^{\prime}\right) \geq 1-x+\frac{c}{2}(1-\ell n 2) \geq \min \left\{\frac{1-\ln 2}{9-\ln 2}, \frac{1-\ln 2}{9-\ell n 2}\right\}=\frac{1-\ln 2}{9-\ln 2} .
$$

THEOREM 1. For each $\alpha$ and $c$, where $0 \leq \alpha<1$ and $0 \leq c \leq 1, \psi_{c}$ has a fixed point satisfying (7a).

Proof. Note, via Lemma 2(iii), that there exists a positive constant $\tilde{m}$ such that

$$
\max \left\{\max _{\left(k_{1}, c, \alpha\right) \in \bar{S}_{\alpha}}\left(1-a_{1}+b_{1}\right), \max _{\left(k_{2}, c, \alpha\right) \in \bar{S}_{\alpha}}\left(1-a_{2}+b_{2}\right)\right\} \leq \tilde{m} .
$$

Choose $a=\min \left(\frac{1}{2}, \frac{m}{2}\right)$ and $b=\frac{1}{m}+\tilde{m}+1$, where $m$ is chosen as in Lemma 5 . Set $D=\left\{\left(K_{1}, K_{2}\right) \in C[-\alpha, 1] \times C[\alpha, 1]: a<K_{1}(\mu), K_{2}\left(\mu^{\prime}\right)<b\right.$ for all $\left(\mu, \mu^{\prime}\right) \in$ $[-\alpha, 1] \times[\alpha, 1]\}$. Clearly, $D$ is a nonempty bounded open subset of $C[-\alpha, 1] \times C[\alpha, 1]$, and $\psi_{c}: \bar{D} \rightarrow C[-\alpha, 1] \times C[\alpha, 1]$ is compact. Next, we show that if $\left(K_{1}, K_{2}\right)=$ 
$\psi_{c}\left(K_{1}, K_{2}\right)$ for $\left(K_{1}, K_{2}\right) \in \bar{D}$, then $\left(K_{1}, K_{2}\right) \in D$. To prove this note first that from the a priori bound for $\left(K_{1}, K_{2}\right)$, we see that $K_{1}(\mu), K_{2}\left(\mu^{\prime}\right) \geq m>a$ for all $\mu, \mu^{\prime}$. Second,

$$
\left\|\left(K_{1}, K_{2}\right)\right\|_{\infty}=\left\|\psi_{c}\left(K_{1}, K_{2}\right)\right\|_{\infty} \leq \tilde{m}+\frac{c(1+\alpha)}{2 m}<b .
$$

Thus $u \in D$ as asserted. The preparations for the use of degree are now complete. Consider the homotopy $I-\psi_{c}$. By homotopy invariance (see, e.g., Theorem 13.6 of $[9])$, since $(1,1) \in D$,

$$
d\left(I-\psi_{c},(0,0), D\right)=d\left(I-\psi_{0},(0,0), D\right)=d(I,(1,1), D)=1 .
$$

Therefore, the existence of equation (1) now follows from the Leray-Schauder fixed point theorem. lemma.

To show the uniqueness of equation (1) satisfying (7a), we need the following

LEMMA 6. Equation (1) has minimal positive solutions $H_{1, \min }(\mu)$ and $H_{2, \min }\left(\mu^{\prime}\right)$ in the following sense : if $H_{1}(\mu)$ and $H_{2}\left(\mu^{\prime}\right)$ are positive solutions of $(1)$, then $H_{1, \min }(\mu) \leq H_{1}(\mu)$ and $H_{2, \min }\left(\mu^{\prime}\right) \leq H_{2}\left(\mu^{\prime}\right)$ for all $\mu, \mu^{\prime}$.

Proof. Consider the two iterates $\left\{H_{1}^{(p)}\right\}$ and $\left\{H_{2}^{(p)}\right\}$ defined as follows:

$$
\begin{gathered}
H_{1}^{(1)}(\mu)=1 \\
H_{2}^{(1)}\left(\mu^{\prime}\right)=1 \text { for all } \mu, \mu^{\prime} \\
H_{1}^{(p+1)}(\mu)=1+\frac{c}{2} H_{1}^{(p)}(\mu)(\mu+\alpha) \int_{\alpha}^{1} \frac{H_{2}^{(p)}\left(\mu^{\prime \prime}\right)}{\mu+\mu^{\prime \prime}} d \mu^{\prime \prime}
\end{gathered}
$$

and

$$
H_{2}^{(p+1)}\left(\mu^{\prime}\right)=1+\frac{c}{2} H_{2}^{(p)}\left(\mu^{\prime}\right)\left(\mu^{\prime}-\alpha\right) \int_{-\alpha}^{1} \frac{H_{1}^{(p)}\left(\mu^{\prime \prime}\right)}{\mu^{\prime}+\mu^{\prime \prime}} d \mu^{\prime \prime} .
$$

Clearly, for each $\mu$ and $\mu^{\prime},\left\{H_{1}^{(p)}(\mu)\right\}$ and $\left\{H_{2}^{(p)}\left(\mu^{\prime}\right)\right\}$ are both increasing sequences. It follows from Theorem 1 that equation (1) has positive solutions, say $H_{1}(\mu)$ and $H_{2}\left(\mu^{\prime}\right)$. Since $H_{1}(\mu) \geq 1$ and $H_{2}\left(\mu^{\prime}\right) \geq 1$ for all $\mu, \mu^{\prime}$, an easy induction would yield $H_{1}^{(p)}(\mu) \leq H_{1}(\mu)$ and $H_{2}^{(p)}\left(\mu^{\prime}\right) \leq H_{1}\left(\mu^{\prime}\right)$ for all $\mu, \mu^{\prime}$ and all $p$. Hence, the sequences $\left\{H_{1}^{(p)}(\mu)\right\}$ and $\left\{H_{2}^{(p)}\left(\mu^{\prime}\right)\right\}$, respectively, converge upward to two limits, say $\bar{H}_{1}(\mu)$ and $\bar{H}_{2}\left(\mu^{\prime}\right)$. It then follows from the monotone convergence theorem that $\bar{H}_{1}$ and $\bar{H}_{2}$ solve equation (1), and that $\bar{H}_{1}(\mu) \leq H_{1}(\mu)$ and $\bar{H}_{2}\left(\mu^{\prime}\right) \leq H_{2}\left(\mu^{\prime}\right)$ for all $\mu, \mu^{\prime}$. The proof of the lemma is thus complete.

TheOREM 2. For $c=0$ or $\alpha=1$, equation (1) has unique solutions. Furthermore, for $0<c<1$, equation (1) has unique solutions $H_{1}$ and $H_{2}$ satisfying (7a).

Proof. The uniqueness for $c=0$ or $\alpha=1$ is trivial. For $0<c<1$, and $1-x>0$ and $1-y>0$, we have that

$$
\begin{aligned}
& 1-x_{\min }:=1-\frac{c}{2} \int_{-\alpha}^{1} H_{1, \min }(\mu) d \mu \geq 1-x>0, \\
& 1-y_{\min }:=1-\frac{c}{2} \int_{\alpha}^{1} H_{2, \min }\left(\mu^{\prime}\right) d \mu^{\prime} \geq 1-y>0
\end{aligned}
$$


and

$$
\begin{aligned}
{\left[H_{2}\left(\mu^{\prime}\right)\right]^{-1} } & =\frac{1-c}{1-y}+\frac{c}{2} \int_{-\alpha}^{1} \frac{\mu^{\prime \prime}+\alpha}{\mu^{\prime}+\mu^{\prime \prime}} H_{1}\left(\mu^{\prime \prime}\right) d \mu^{\prime \prime} \\
& \geq \frac{1-c}{1-y_{\min }}+\frac{c}{2} \int_{-\alpha}^{1} \frac{\mu^{\prime \prime}+\alpha}{\mu^{\prime}+\mu^{\prime \prime}} H_{1, \min }\left(\mu^{\prime \prime}\right) d \mu^{\prime \prime} \\
& \geq\left[H_{2, \min }\left(\mu^{\prime}\right)\right]^{-1} .
\end{aligned}
$$

Therefore, $H_{2}=H_{2, \min }$, and hence $H_{1}=H_{1, \min }$, and the lemma is proved.

Our final result is concerned with the number of positive solutions for equation (1). The techniques for proving this result are motivated by those of Leggett [10]. To this end, we first prove the following lemma.

LEMMA 7. Let $0<c<1$ and $0 \leq \alpha<1$, and let $\left(H_{1}, H_{2}\right)$ and $\left(\bar{H}_{1}, \bar{H}_{2}\right)$ be positive solutions pairs of equation (1) satisfying $(7 \mathrm{a})$ and $(7 \mathrm{~b})$, respectively. Then the following holds:

(i) There exist, respectively, two positive constants $k_{1}$ and $k_{2}$, where $0<k_{1}<\frac{1}{1+\alpha}$ and $0<k_{2}<\frac{1}{1-\alpha}$, such that

$$
\frac{c}{2} \int_{-\alpha}^{1} \frac{H_{1}\left(\mu^{\prime \prime}\right)}{1-k_{1}\left(\mu^{\prime \prime}+\alpha\right)} d \mu^{\prime \prime}=1
$$

and

$$
\frac{c}{2} \int_{\alpha}^{1} \frac{H_{2}\left(\mu^{\prime \prime}\right)}{1-k_{2}\left(\mu^{\prime \prime}-\alpha\right)} d \mu^{\prime \prime}=1 .
$$

Furthermore, such choices of $k_{1}$ and $k_{2}$ are unique.

(ii) There exist, respectively, two positive constants $\bar{k}_{1}$ and $\bar{k}_{2}$, where $0<\bar{k}_{1}<\frac{1}{1+\alpha}$ and $0<\bar{k}_{2}<\frac{1}{1-\alpha}$, such that

$$
\frac{c}{2} \int_{-\alpha}^{1} \frac{\bar{H}_{1}\left(\mu^{\prime \prime}\right)}{1+\bar{k}_{2}\left(\mu^{\prime \prime}+\alpha\right)} d \mu^{\prime \prime}=1
$$

and

$$
\frac{c}{2} \int_{\alpha}^{1} \frac{\bar{H}_{2}\left(\mu^{\prime \prime}\right)}{1+\bar{k}_{1}\left(\mu^{\prime \prime}-\alpha\right)} d \mu^{\prime \prime}=1 .
$$

Moreover, such choices of $\bar{k}_{1}$ and $\bar{k}_{2}$ are unique.

Proof. Since the analysis leading to (10a), (10b), (10c), and (10d) is similar, we illustrate only (10b) and (10c). Define the function $T:\left(0, \frac{1}{1-\alpha}\right) \rightarrow R$ by

$$
T(k)=\frac{c}{2} \int_{\alpha}^{1} \frac{H_{2}\left(\mu^{\prime \prime}\right)}{1-k\left(\mu^{\prime \prime}-\alpha\right)} d \mu^{\prime \prime} .
$$

Then

$$
\lim _{k \rightarrow\left(\frac{1}{1-\alpha}\right)^{-}} T(k)=\frac{c}{2} \int_{\alpha}^{1} \frac{(1-\alpha) H_{2}\left(\mu^{\prime \prime}\right)}{1-\mu^{\prime \prime}} d \mu^{\prime \prime},
$$

since $\left(1-k\left(\mu^{\prime \prime}-\alpha\right)\right)^{-1}$ increases monotonically with $k, 0<k<\frac{1}{1-\alpha}$. Note that the improper integral in (11) diverges to $+\infty$. Since $T(0)=\frac{c}{2} \int_{\alpha}^{1} H_{2}\left(\mu^{\prime \prime}\right) d \mu^{\prime \prime}<1$, and 
since $T(k)$ is strictly increasing with $T\left(\frac{1}{1-\alpha}\right)=+\infty$, there exists a unique $k_{2} \in\left(0, \frac{1}{1-\alpha}\right)$ for which (10b) holds. Now suppose that $\bar{H}_{1}$ and $\bar{H}_{2}$ satisfy (1) and (7b). Then $\frac{c}{2} \int_{-\alpha}^{1} \bar{H}_{1}\left(\mu^{\prime \prime}\right) d \mu^{\prime \prime}>1$, and

$$
\begin{aligned}
\frac{c}{2} \int_{-\alpha}^{1} \frac{\bar{H}_{1}\left(\mu^{\prime \prime}\right)}{1+\frac{1}{1-\alpha}\left(\mu^{\prime \prime}+\alpha\right)} d \mu^{\prime \prime} & =\frac{c}{2} \int_{-\alpha}^{1} \frac{1-\alpha}{1+\mu^{\prime \prime}} \bar{H}_{1}\left(\mu^{\prime \prime}\right) d \mu^{\prime \prime} \\
& =1-\left[\bar{H}_{2}(1)\right]^{-1}<1
\end{aligned}
$$

Therefore, there exists a unique $\bar{k}_{2}, 0<\bar{k}_{2}<\frac{1}{1-\alpha}$, such that (10c) holds.

THEOREM 3. Equation (1) has exactly two positive solutions if $0<c<1$ and $0 \leq \alpha<1$.

Proof. Let $H_{1}$ and $H_{2}$ be positive solutions of (1) satisfying (7a). Define

$$
\bar{H}_{1}(\mu)=\frac{1+k_{2} \mu+k_{2} \alpha}{1-k_{1} \mu-k_{1} \alpha} H_{1}(\mu)
$$

and

$$
\bar{H}_{2}\left(\mu^{\prime \prime}\right)=\frac{1+k_{1} \mu^{\prime \prime}-k_{1} \alpha}{1-k_{2} \mu^{\prime \prime}+k_{2} \alpha} H_{2}\left(\mu^{\prime \prime}\right) .
$$

Here $k_{1}$ and $k_{2}$ are chosen as in Lemma 7. Now, using (10b), we find

$$
\begin{aligned}
\frac{c}{2} \int_{\alpha}^{1} \frac{\mu+\alpha}{\mu+\mu^{\prime \prime}} \cdot \bar{H}_{2}\left(\mu^{\prime \prime}\right) d \mu^{\prime \prime}= & \frac{c}{2} \int_{\alpha}^{1} \frac{(\mu+\alpha)\left(1+k_{1} \mu^{\prime \prime}-k_{1} \alpha\right)}{\left(\mu+\mu^{\prime \prime}\right)\left(1-k_{2} \mu^{\prime \prime}+k_{2} \alpha\right)} H_{2}\left(\mu^{\prime \prime}\right) d \mu^{\prime \prime} \\
= & \frac{1-k_{1} \mu-k_{1} \alpha}{1+k_{2} \mu+k_{2} \alpha}\left(\frac{c}{2}\right) \int_{\alpha}^{1} \frac{\mu+\alpha}{\mu+\mu^{\prime \prime}} H_{2}\left(\mu^{\prime \prime}\right) d \mu^{\prime \prime} \\
& +\frac{\left(k_{1}+k_{2}\right)(\mu+\alpha)}{1+k_{2} \mu+k_{2} \alpha}\left(\frac{c}{2}\right) \int_{\alpha}^{1} \frac{H_{2}\left(\mu^{\prime \prime}\right)}{1-k_{2} \mu^{\prime \prime}+k_{2} \alpha} d \mu^{\prime \prime} \\
= & \frac{1-k_{1} \mu-k_{1} \alpha}{1+k_{2} \mu+k_{2} \alpha}\left[1-\frac{1}{H_{1}(\mu)}\right]+\frac{\left(k_{1}+k_{2}\right)(\mu+\alpha)}{1+k_{2} \mu+k_{2} \alpha} \\
= & 1-\frac{1}{\bar{H}_{1}(\mu)} .
\end{aligned}
$$

A similar computation would yield that

$$
\frac{c}{2} \int_{-\alpha}^{1} \frac{\mu^{\prime}-\alpha}{\mu^{\prime}+\mu^{\prime \prime}} \bar{H}_{1}\left(\mu^{\prime \prime}\right) d \mu^{\prime \prime}=1-\frac{1}{\bar{H}_{2}\left(\mu^{\prime}\right)} .
$$

That is, $\bar{H}_{1}$ and $\bar{H}_{2}$ satisfy equation (1). Hence, $\bar{H}_{1}$ and $\bar{H}_{2}$ must satisfy either (7a) or (7b). Since $H_{1}$ and $H_{2}$ are the unique positive solutions of (1) satisfying (7a), and since $\bar{H}_{1}(\mu)>H_{1}(\mu), \bar{H}_{2}\left(\mu^{\prime}\right)>H_{2}\left(\mu^{\prime}\right)$ for almost all $\mu, \mu^{\prime}, \bar{H}_{1}$ and $\bar{H}_{2}$ must satisfy (7b). Thus, we have shown that equation (1) has at least two positive solutions when $c$ and $\alpha$ are as assumed. It remains to show that such an equation has at most two 
solutions. To this end, we suppose that $\bar{H}_{1}$ and $\bar{H}_{2}$ are positive solutions satisfying (1) and (7b). Define

$$
H_{1}(\mu)=\frac{1-\bar{k}_{1} \mu-\bar{k}_{1} \alpha}{1+\bar{k}_{2} \mu+\bar{k}_{2} \alpha} \bar{H}_{1}(\mu)
$$

and

$$
H_{2}\left(\mu^{\prime \prime}\right)=\frac{1-\bar{k}_{2} \mu^{\prime \prime}+\bar{k}_{2} \alpha}{1+\bar{k}_{1} \mu^{\prime \prime}-\bar{k}_{1} \alpha} \bar{H}_{2}\left(\mu^{\prime \prime}\right) .
$$

Here $\bar{k}_{1}$ and $\bar{k}_{2}$ are chosen as in Lemma 7. Now, using (10c), we obtain

$$
\begin{aligned}
\frac{c}{2} \int_{-\alpha}^{1} \frac{\mu^{\prime}-\alpha}{\mu^{\prime}+\mu^{\prime \prime}} \cdot H_{1}\left(\mu^{\prime \prime}\right) d \mu^{\prime \prime}= & \frac{c}{2} \int_{-\alpha}^{1} \frac{\left(\mu^{\prime}-\alpha\right)\left(1-\bar{k}_{1} \mu^{\prime \prime}-\bar{k}_{1} \alpha\right)}{\left(\mu^{\prime}+\mu^{\prime \prime}\right)\left(1+\bar{k}_{2} \mu^{\prime \prime}+\bar{k}_{2} \alpha\right)} \bar{H}_{1}\left(\mu^{\prime \prime}\right) d \mu^{\prime \prime} \\
= & \frac{1+\bar{k}_{1} \mu^{\prime}-\bar{k}_{1} \alpha}{1-\bar{k}_{2} \mu^{\prime}+\bar{k}_{2} \alpha}\left(\frac{c}{2}\right) \int_{-\alpha}^{1} \frac{\mu^{\prime}-\alpha}{\mu^{\prime}+\mu^{\prime \prime}} \bar{H}_{1}\left(\mu^{\prime \prime}\right) d \mu^{\prime \prime} \\
& -\frac{\left(\bar{k}_{1}+\bar{k}_{2}\right)\left(\mu^{\prime}-\alpha\right)}{1-\bar{k}_{2} \mu^{\prime}+\bar{k}_{2} \alpha}\left(\frac{c}{2}\right) \int_{-\alpha}^{1} \frac{\bar{H}_{1}\left(\mu^{\prime \prime}\right)}{1+\bar{k}_{2} \mu^{\prime \prime}+\bar{k}_{2} \alpha} d \mu^{\prime \prime} \\
= & \frac{1+\bar{k}_{1} \mu^{\prime}-\bar{k}_{1} \alpha}{1-\bar{k}_{2} \mu^{\prime}+\bar{k}_{2} \alpha}\left[1-\frac{1}{\bar{H}_{2}\left(\mu^{\prime}\right)}\right]-\frac{\left(\bar{k}_{1}+\bar{k}_{2}\right)\left(\mu^{\prime}-\alpha\right)}{1-\bar{k}_{2} \mu^{\prime}+\bar{k}_{2} \alpha} \\
= & 1-\frac{1}{\bar{H}_{2}\left(\mu^{\prime}\right)} .
\end{aligned}
$$

Similarly, we obtain that

$$
\frac{c}{2} \int_{\alpha}^{1} \frac{\mu+\alpha}{\mu+\mu^{\prime \prime}} H_{2}\left(\mu^{\prime \prime}\right) d \mu^{\prime \prime}=1-\frac{1}{H_{1}(\mu)} .
$$

Therefore, $H_{1}$ and $H_{2}$ satisfy equation (1). It follows from (13) and (10c), (10d) that $\frac{c}{2} \int_{-\alpha}^{1} H_{1}(\mu) d \mu<1$ and $\frac{c}{2} \int_{\alpha}^{1} H_{2}\left(\mu^{\prime}\right) d \mu^{\prime}<1$; i.e., $H_{1}$ and $H_{2}$ satisfy (7a). Since the solutions of (1) satisfying (7a) are unique, we conclude that the solutions of (1) satisfying $(7 \mathrm{~b})$ are also unique, and the theorem is proved.

TheOREM 4. Let $c=1$ and let $\alpha$ be sufficiently close to 1 . Then equation (1) has exactly two positive solutions.

Proof. Let $H_{1}$ and $H_{2}$ be solutions of equation (1) satisfying (7a). It follows from Lemma 5 that $y$ must approach zero as $\alpha$ approaches 1 from the left. Hence if $c=1$ and $\alpha$ is chosen to be sufficiently close to 1 , then $x=1$ and $y<1$. Define $\bar{H}_{1}$ and $\bar{H}_{2}$ as follows:

$$
\bar{H}_{1}(\mu)=\left(1+k_{2} \mu+k_{2} \alpha\right) H_{1}(\mu)
$$

and

$$
\bar{H}_{2}\left(\mu^{\prime \prime}\right)=\frac{H_{2}\left(\mu^{\prime \prime}\right)}{1-k_{2} \mu^{\prime \prime}+k_{2} \alpha},
$$

where $k_{2}$ is uniquely satisfied by (10b). Using a procedure similar to the proof of Theorem 3 , it follows that $\bar{H}_{1}$ and $\bar{H}_{2}$ are positive solutions of (1) satisfying (7b). Since $H_{1} \neq \bar{H}_{1}$ and $H_{2} \neq \bar{H}_{2}$, it remains to show that such an equation has at most 
two positive solutions. Suppose $\bar{H}_{1}$ and $\bar{H}_{2}$ are positive solutions of (1) satisfying (7b). Then either

$$
\bar{x}:=\frac{1}{2} \int_{-\alpha}^{1} \bar{H}_{1}(\mu) d \mu>1 \text { and } \bar{y}:=\frac{1}{2} \int_{\alpha}^{1} \bar{H}_{2}\left(\mu^{\prime}\right) d \mu^{\prime}=1,
$$

or

$$
\bar{x}=1 \text { and } \bar{y}>1 \text {, }
$$

or

$$
\bar{x}=1 \text { and } \bar{y}=1 .
$$

If (14c) held, then $\bar{H}_{1}$ and $\bar{H}_{2}$ would also satisfy (7a), and hence $\bar{y} \rightarrow 0$ as $\alpha \rightarrow 1$, a contradication. Thus, (14c) should be ruled out. If (14b) were the case, then $H_{1}$ and $H_{2}$, defined as in (13a) and (13b), respectively, with $\vec{k}_{1}=0$ and $k_{2}$ satisfying (10c), were positive solutions of (1) satisfying (7a). Since $H_{1} \leq \bar{H}_{1}$ and $H_{1} \neq \bar{H}_{1}$, we see immediately that $x<1$ and $y=1$. This is not possible. Therefore, (14a) must hold.

Define $H_{1}$ and $H_{2}$ as in (13a) and (13b), respectively, with $\bar{k}_{2}=0$ and $\bar{k}_{1}$ satisfying (10d). Then such $H_{1}$ and $H_{2}$ are the positive solutions of (1) satisfying (7a). Now, if we can show that the positive solutions of equation (1) satisfying (7a) are unique, then the proof of the theorem will be complete. To this end, we note, as observed in the first paragraph of the proof, that $x_{\min }$ must be equal to 1 . Therefore, $\int_{-\alpha}^{1}\left(H_{1}(\mu)-\right.$ $\left.H_{1, \min }(\mu)\right) d \mu=0$, and so $H_{1} \equiv H_{1, \min }$ and $H_{2} \equiv H_{2, \min }$. Thus, the theorem is proved.

We conclude this paper with the following remarks.

Remarks. 1. We may conclude, via the proofs of Theorems 3 and 4 , that for $c=1$, if $x$ and $y$ are not both equal to 1 , then equation (1) admits exactly two positive solutions.

2. On the other hand, if $x=y=1$, then equation (1) has unique positive solutions. To see this, we note that either $x_{\min }:=\frac{1}{2} \int_{\alpha}^{1} H_{1, \min }(\mu) d \mu$ or $y_{\min }:=$ $\frac{1}{2} \int_{-\alpha}^{1} H_{2, \min }\left(\mu^{\prime}\right) d \mu^{\prime}$ is equal to 1 . We assume, without loss of generality, that $y_{\min }=1$. Thus,

$$
0=\frac{1}{2} \int_{-\alpha}^{1} H_{2, \min }\left(\mu^{\prime}\right)-H_{2}\left(\mu^{\prime}\right) d \mu^{\prime} .
$$

Since $H_{2, \min }-H_{2}$ is a continuous nonpositive function, we find that $H_{2, \min } \equiv H_{2}$, and hence $H_{1, \min } \equiv H_{1}$. Note that for $\alpha=0$ and $c=1$, we have $x=y=1$.

3 . The case where $c$ is not a constant can be easily generalized.

Acknowledgments. The author would like to thank the referees for their helpful comments.

\section{REFERENCES}

[1] I. W. Busbridge, On the H-function of Chandrasekhar, Quart. J. Math. Oxford Ser., 8 (1957), pp. 133-140.

[2] — On solutions of Chandrasekhar's integral equation, Trans. Amer. Math. Soc., 105 (1962), pp. 112-117.

[3] S. Chandrasekhar, Radiative Transfer, Dover, New York, 1960.

[4] — The transfer of radiation in stellar atmospheres, Bull. Amer. Math. Soc., 53 (1947), pp. 641-711. 
[5] F. CoRon, Computation of the asymptotic states for linear half space kinetic problem, Transport Theory Statist. Phys., 19 (1990), pp. 89-114.

[6] M. M. CRUM, On an integral equation of Chandrasekhar, Quart. J. Math. Oxford Ser., 18 (1947), pp. 244-252.

[7] C. Fox, A solution of Chandrasekhar's integral equation, Trans. Amer. Math. Soc., 99 (1961), pp. 285-291.

[8] B. D. Ganapol, An investigation of a simple transport model, Transport Theory Statist. Phys., 21 (1992), pp. 1-37.

[9] V. Huston AND J. S. PyM, Applications of Functional Analysis and Operator Theory, Academic Press, New York, 1980.

[10] R. W. LeggetT, A new approach to the H-equation of Chandrasekhar, SIAM J. Math. Anal., 7 (1976), pp. 542-550.

[11] C. A. StUART, Existence theorems for a class of nonlinear integral equaitons, Math. Z., 137 (1974), pp. 49-66. 